\title{
An Improved Cellular Automata Model Considering Driver Types
}

\author{
Dan Wang ${ }^{a}$ and Beilei $\mathrm{Li}^{\mathrm{b}}$ \\ Key Laboratory of Manufacturing Industrial Integrated Automation, Shenyang University, \\ Shenyang, China \\ a48112951@qq.com, b15840109143@163.com
}

Keywords: Traffic flow, Cellular automata.

Abstract. Based on the classical NS and FI model, an improved cellular automata model is proposed taking into account the different types of drivers. The drivers are divided into the energetic and conservative. Moreover, the evolution and update rules are given for different vehicles considering their respective driving preference. Numerical simulations show that the traffic flow rate is dramatically increased on the new model, and the threshold of the road capacity is greater increased than ever.

\section{Introduction}

In recent years, with the rapid development of information revolution, the megalopolis appeared "Pan City" phenomenon, the traffic problems and road congestion problems have become increasingly prominent, and this will undoubtedly increase the burden of city traffic. Traffic flow theory is to explain and analyze traffic problems and guidance based on traffic management, traffic phenomenon, and it can effectively guide the traffic congestion problem solving [1].

Microscopic traffic flow model of vehicle is widely used in traffic flow modeling. In 1983, the famous 184 cellular automata model was proposed [2], in this model, the road was divided into isometric grid, each grid point represents a cellular model, and each cell state is either empty or occupied by a car. In 1992, NS model based on 184 model is proposed, which can simulate freeway traffic cellular automaton [3]. NS model is a one-dimensional single lane cellular traffic flow model (CA model). Then, the Japanese scholars Fukui and Ishibashi proposed the FI model [4]. In this model, if the speed between the vehicle and in front spacing is greater than the maximum speed of vehicle, the vehicle will move forward with maximum speed, while the speed between the vehicle and in front spacing is less than the maximum speed of vehicle, the vehicle forward speed equal to primary speed [5]. In this paper, we consider a cellular automata traffic flow model based on driver's liquidity preference from the point of view of psychology and behavior, and the drivers are divided into energetic and conservative. In the periodic boundary conditions according to their preferences for different vehicle and update rules, and at the traffic lights junction of the car that slow start behavior is added to the new model, which makes the model more realistic traffic conditions.

\section{The Traffic Flow Model}

In a previous study is often used NS model and FI model, the NS model is a stochastic CA model of traffic flow, each vehicle state by the speed and position of the said, the state in accordance with the following rules are updated in parallel:

a) the acceleration process: $V_{n} \rightarrow \min \left(V_{n+1}, V_{\max }\right)$

b) safety braking process: $V_{n} \rightarrow \min \left(V_{n}, d_{n-1}\right)$

c)randomization slowing down process: $V_{n} \rightarrow \max \left(V_{n}-1,0\right)$

d) location update: $X_{n} \rightarrow X_{n}+V_{n}$ 
As same as the emergence of obstruction point, which the average speed was $150 \mathrm{~km} / \mathrm{h}$. But the maximum flow is not the same as in Figure 1, which is $25 \%$ lower than the measured data with the resulting NS model. The reason is that the relative motion of vehicle, considering the relative speed makes the motion of the vehicle speed and traffic flow increased. But in the FI model, there are some different cases. According to the random deceleration rule, when the vehicle is able to maximum speed, it will with probability P from Vmax to Vmax-1. The difference between FI model and NS model is accelerated and the random deceleration in different ways. In the NS model, if the vehicle speed is less than the maximum speed, then speed of vehicles in the next time will up to $\mathrm{V}+1$, but in the FI model, as long as the actual speed is less than the maximum speed of the vehicle, may be in the next time step to accelerate to the maximum speed (considering random rule); in NS model, all the vehicles may be the random deceleration, and in the FI model, only running at maximum speed has to decelerate. On the basis of theory of NS model and FI model, we consider two type of driver: energetic and conservative. For the dynamic type driver, driving behavior more radical, with high liquidity as his driving goal, is a type of liquidity preference is very strong, which more suitable for FI cellular automata traffic flow model, as long as the speed is less than the maximum speed, the vehicle could accelerate to the maximum speed (considering random rules). Because in the real traffic environment, on the one hand, the leading vehicle rarely used "wall" brake, bringing about the driver to the adventure travel style of driving, which caused some problems for traffic safety as possible collision accident. Through the above analysis we can see that the actual traffic flow is that there are two types of drivers -- the vitality and conservative.

\section{Driver Type}

\section{Energetic Type}

Based on the above analysis, update rules for vitality type driver can be used as follows (considering the effects of spacing for driving the vehicle speed):

a) The acceleration process:

$$
V_{i}\left(t+\frac{1}{2}\right) \Rightarrow \min \left\{\mathrm{F}\left[d_{i}(t)\right], V_{\max }\right\}
$$

Among them, $\mathrm{Vi}(\mathrm{t})$ represents the speed at the moment of No.i vehicle; di $(\mathrm{t})$ represents the distance between the leading vehicle and the No.i vehicle at time $\mathrm{t}, \mathrm{F}$ is a function of the speed about distance. b) Safety deceleration process: This process occurs near the traffic lights at the junction position and the leading vehicle's slow start phenomenon when vehicle traffic signals got.

If $V_{i+1}(t)=0$, the leading car start slowing down the speed is equal to 0 , dynamic type driver can be used :

$$
V_{i}(\mathrm{t}+1)=\left\{\begin{array}{l}
\max \left\{\min \left(V_{i}\left(\mathrm{t}+\frac{1}{2}\right), \mathrm{F}\left[d_{i}(t)\right]-1,0\right)\right\} \\
\min \left\{V_{i}\left(\mathrm{t}+\frac{1}{2}\right), \mathrm{F}\left[d_{i}(t)\right]\right\}
\end{array}\right\}
$$

c) Deterministic reduction process: If $V_{i+1}(t) !=0$, the vehicle speed is not 0. For the dynamic type driver, will not give up the opportunity make headway speed which shorten the distance with the leading vehicle. Therefore:

$$
V_{i}(\mathrm{t}+1)=V_{i}\left(t+\frac{1}{2}\right)
$$

d) Location update:

$$
X_{i}(\mathrm{t}+1)=X_{i}(\mathrm{t})+V_{i}(\mathrm{t}+1)
$$




\section{Conservative Type}

However, actual traffic environment consisted of a dynamic type driver and conservative driver together, we make update rule for conservative drivers below:

a)The acceleration process: Conservative driver in the probability of the process may be accelerated slants small which try to keep a larger safe distance from the leading vehicle, the acceleration process is relatively long, the speed is relatively low, therefore, can be used:

$$
V_{i}\left(\mathrm{t}+\frac{1}{2}\right)=\min \left(V_{i}(\mathrm{t})+1, V_{\max }\right)
$$

b) Randomization slowing down process: All vehicles on the road (assuming conservative and vigor) have a certain probability of $\mathrm{P}$ random deceleration, the speed less 1 on the basis of the original, can be used:

$$
V_{i}\left(t+\frac{1}{2}\right)=\left\{\max \left\{V_{i}\left(t+\frac{1}{2}\right)-1,0\right\}\right\}
$$

c) Safety deceleration process: This process occurs near the traffic lights at the junction position and the leading vehicle's slow start phenomenon when vehicle traffic signals got.

If $V_{i+1}(t)=0$, the leading car start slowing down the speed is equal to 0 , conservative type driver can be used :

$$
V_{i}(\mathrm{t}+1)=\left\{\begin{array}{l}
\max \left\{\min \left(V_{i}\left(\mathrm{t}+\frac{1}{2}\right), \mathrm{F}\left[d_{i}(t)\right]-1,0\right)\right\} \\
\min \left\{V_{i}\left(\mathrm{t}+\frac{1}{2}\right), \mathrm{F}\left[d_{i}(t)\right]\right\}
\end{array}\right\}
$$

d) Deterministic reduction process: Generally occurs after the random deceleration or safety deceleration behavior (conservative driver for this), which is a known speed and time relationship, making a judgment on the deceleration in advance, can be used:

If $V_{i+1}(t) !=0$, the vehicle speed is not 0 . For the conservative type driver can be used:

$$
V_{i}(\mathrm{t}+1)=\min \left\{V_{i}\left(\mathrm{t}+\frac{1}{2}\right), \mathrm{F}\left[d_{i}(t)\right]\right\}
$$

e) Location update: for the conservative type driver can be used:

$$
X_{i}(\mathrm{t}+1)=X_{i}(\mathrm{t})+V_{i}(\mathrm{t}+1)
$$

In this new model, a mixed traffic flow model, we rehard the conservative driver as cellular following status, using some characteristics in the car following model, the vehicle state stochastic delay theory is a kind of WWH model evolution update rule. But for the dynamic type driver is a different situation, these people like to drive fast, look upon the vehicle flow ability as the goal and the pursuit of efficiency. They less on the brake, vehicle spacing is not great. Based on this, in the new model, dynamic type driver prefer to use FI acceleration mechanism, considering the multi speed changes have the potential to reach the maximum value, which will be more in line with the complicated traffic flow problems.

\section{Numerical simulations}

We will use the length of $\mathrm{L}$ for the $7.5 \mathrm{~m}$ lattice to represent a single lane segment, the maximum speed of actual $135 \mathrm{~km} / \mathrm{h}$ with periodic boundary conditions. In the initial state, we assume that the dynamic type driver with the conservative driver respectively with different proportion in the system means $\mathrm{N}$ cellular, using Pd and Pc to represent the number of vehicles. Vehicle density is $\rho=\frac{N}{L}$ and traffic flow is $Q(\mathrm{t})=\rho \bar{v}(\mathrm{t})$. 


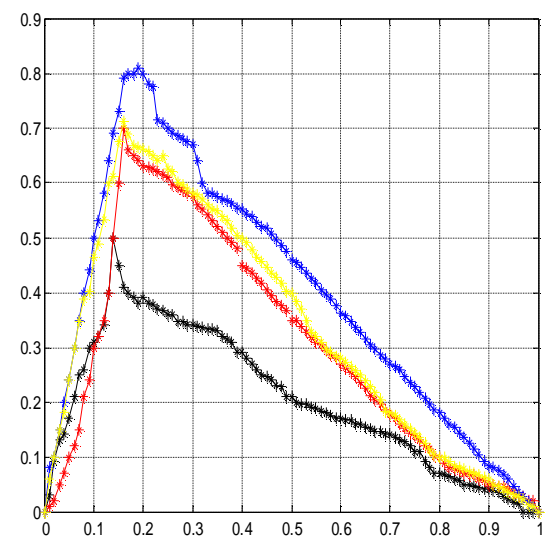

Fig 1 The traffic flow and density in different mixing ratio between two drivers

In Fig. 1, we use four different colors of the curves representing the four sets of data, the black represents the first group, namely $\mathrm{Pe}=0.0 \mathrm{Pc}=1.0$; the red represents second groups, namely $\mathrm{Pe}=0.2$ $\mathrm{Pc}=0.8$; the blue on behalf of third groups, namely $\mathrm{Pe}=0.5 \mathrm{Pc}=0.5$; the yellow for the fourth groups, namely $\mathrm{Pe}=1.0 \mathrm{Pc}=0$. It can be seen from Fig. 1 , the average rate of 3 group and 4 group can reach a maximum of $138 \mathrm{~km} / \mathrm{h}-1$, while the average speed of the 1 group to a maximum of $124 \mathrm{~km} / \mathrm{h}-1$. The reason is that these two types drivers have different driving style. That is to say, two types of vehicle acceleration mechanism and the random deceleration mechanism is different. The energetic type in every time step can speed up to the maximum speed, the vehicle will be in the random deceleration probability pA reduction and only when vehicle spacing is less than Vmax. The conservative type vehicle each time step speeds up to 1 , and will be from any speed of $V \in(0, \mathrm{Vmax})$, with the random deceleration probability PC down to V-1. So in the low density area, after a period of time, the system will run smoothly at high speed state, the distance between the car become larger. In view of this, the energy driver need not the random deceleration, speed can reach a maximum value during this period. But the conservative driver need declare speed and increase with the random deceleration, the speed will continue to fall.

\section{Acknowledgements}

This work was financially supported by National Nature Science Foundation (61203152), Program for Liaoning Excellent Talents in University (LNET) (LJQ2013122) and Natural Science Foundation of Liaoning Province (Grant No. 2015020037).

\section{References}

[1] S. Wolfram, Theory and Application of Cellular Automata(World Scientific,Singapore,1986)

[2] M R Liu, Y.Xue, L J Kong., City Road Traffic And Traffic Flow Models , Mechanics and Practice, , 27(1):1-6, 2005.

[3] S. Wolfram., Statistical mechanics of cellular automata, Rev. Mod. Phys.55 601-644, 1983.

[4] K.Nagel. M.Schreckenberg, A Cellular Automaton Model for Freeway Traffic, Journal de Physique I,2(12): 2211-2229,1992.

[5] M.Fukui., Y.Ishibashi, Traffic Flow in 1D Cellular Automata Model In-cluding CarsMoving with High Speed [J]1Japan: J1Phys1Soc1,65(1):868-8701.1996. 